\title{
Copper $\mathbf{N}$-heterocyclic carbene complexes as active catalysts for the
}

\section{synthesis of 2-substituted oxazolines from nitriles and aminoalco-}

\section{hols}

\author{
Michael Trose, Faïma Lazreg, Mathieu Lesieur and Catherine S. J. Cazin*
}

EaStCHEM School of Chemistry, University of St Andrews, St Andrews, KY16 9ST, UK.

\begin{abstract}
The reaction between nitriles and aminoalcohols to access 2-substituted oxazolines was investigated. Using copper-NHC complexes, various nitriles were successfully converted into the corresponding oxazolines, under milder and less wasteful conditions than previously reported methods.
\end{abstract}

\section{INTRODUCTION}

2-Subtituted oxazolines constitute the core structure of many biologically active natural compounds and play a major role in medicinal chemistry. ${ }^{1}$ Regarding their applications, these molecules are well-known ligands for transition metals in homogenous catalysis, in particular for the synthesis of chiral products. ${ }^{2}$ In organic synthesis, oxazolines are common synthetic intermediates ${ }^{3}$ and can also be used as protecting groups for carboxylic acids due to their resistance toward nucleophiles, bases, radicals, and even some acids. ${ }^{4}$ Considering their broad applicability, several protocols have been developed to synthetize this important class of compounds. ${ }^{5}$ However, the widespread synthetic methodologies leading to this important class of compounds involve cyclodehydration of $\beta$-hydroxy amides ${ }^{6}$ or the condensation of carbonyls ${ }^{7}$ or nitriles with aminoalcohols. Among these options, nitriles have been widely studied because they are readily available and inexpensive starting materials. The common approach requires an electrophile to activate a nitrile (1) for a nucleophilic attack by an aminoalcohol (2) (Scheme 1). This transformation can be achieved using heterogeneous catalysts such as silica sulfuric acid (SSA) $)^{8}$ or tungstophosphoric acid (TPA). ${ }^{9}$ Different metal salts have also been tested for this purpose. Mohammadpoor-Baltork and co-workers developed two procedures using $\operatorname{Bi}(\mathrm{III})^{10}$ or $\operatorname{Zr}(\mathrm{IV})^{11}$ salts; however, despite the good functional group tolerance of these protocols, a large excess of aminoalcohol (up to 8 equivalents) is needed to achieve good isolated yields. Recently, Ge and co-workers have used a catalytic amount of $\mathrm{Co}\left(\mathrm{NO}_{3}\right)_{2}$ to synthesize 2- 
subtituted oxazolines, ${ }^{12}$ with elemental sulfur added as a nitrile activator. Moreover, Fu and co-workers reported that $\mathrm{Zn}(\mathrm{OTf})_{2}$ in toluene at reflux is an active catalyst for the synthesis of chiral pyridine bis(oxazoline) ligands. ${ }^{13}$ Similarly, well-defined organometallic complexes have also been tested as catalysts for this transformation, especially copper, due to its abundance, low toxicity and affordability with respect to other metals, such as Pd, Pt or Ru. Li and co-workers developed a methodology using a catalytic amount of a $\mathrm{Cu}_{2}{ }_{2} \mathrm{~L}_{4}(\mathrm{~L}=$ methacrylate $)$ complex. ${ }^{14}$ Unfortunately, a relatively high catalyst loading ( $8 \mathrm{~mol} \%)$ and an excess of base ( 2 equiv. of $\mathrm{NaOAc}$ ) were required, as well as the need for the independent preparation of the $\mathrm{Cu}_{2}{ }_{2} \mathrm{~L}_{4}$ catalyst. Similar drawbacks were observed with the use of copper(II) pyrazolate complexes, with 4 equiv. of aminoalcohol and $8 \mathrm{~mol} \%$ of catalyst being required. ${ }^{15}$

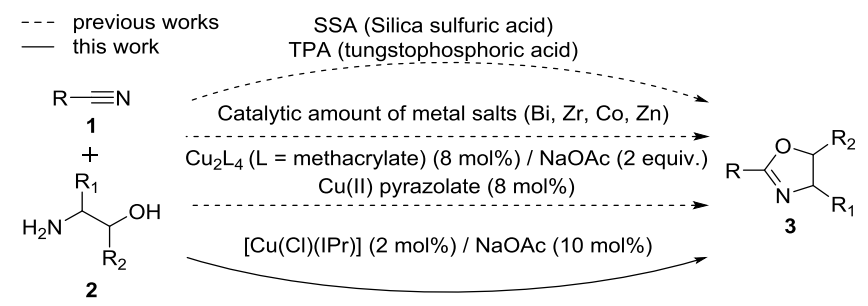

\section{Scheme 1. Synthesis of 2-substituted oxazolines from nitriles and aminoalcohols}

The most frequently encountered drawbacks for all the aforementioned procedures are the large excesses of 2 (4-8 equiv.) or additives needed to achieve the desired products. Considering these disadvantages, a methodology that can minimize these amounts of reactants is highly desirable. In the last decade, well-defined copper $N$-heterocyclic carbene (NHC) complexes have emerged as active catalysts in a plethora of reactions that include $[3+2]$ cycloaddition, ${ }^{16}$ borylation of alkynes, ${ }^{17}$ synthesis of phenols ${ }^{18}$ and many others. ${ }^{19}$ Up to now, the use of these complexes as catalysts in the formation of oxazoline derivatives has not been reported. Herein, a copper-NHC catalyzed procedure has been developed in which only a substoichiometric amount of base and a small excess of aminoalcohol is used.

\section{RESULTS AND DISCUSSION}

Recently, our group reported a straightforward procedure for the synthesis of 2-substituted thiazolines using only a catalytic amount of $\mathrm{NaOH} .{ }^{20}$ While trying to expand this base-catalyzed protocol to the synthesis of 2-substituted oxazolines, using benzonitrile $\mathbf{1 a}$ and ethanolamine $\mathbf{2 a}$ as model substrates, we found that a catalytic amount of the base (10 mol\%) was solely capable to promote this reaction (Table 1). Strong bases, such as alkali hydroxides (Table 1, entries 2 and 3 ) and tert-butoxides (Table 1, entry 4), gave only poor conversions, whereas slightly better results were obtained using weak bases, especially $\mathrm{Na}_{2} \mathrm{CO}_{3}$ and NaOAc (Table 1, entries 6 and 9, respectively). This observation is in accordance with previous work using an excess of the same weak bases. ${ }^{5 e, 14}$ Using the base alone as catalyst, the amount of $2 \mathbf{a}$ was found to be 
crucial. In the presence of $\mathrm{Na}_{2} \mathrm{CO}_{3}$, increasing the amount of $2 \mathrm{a}$ leads to a drop in conversion (Table 1, entries 6-8) while an opposite effect is witnessed when using NaOAc (Table 1, entries 9-11).

Table 1. Screening of bases for the synthesis of 2-phenyl-4,5-dihydrooxazole ${ }^{a}$

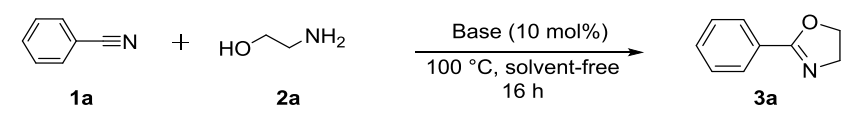

\begin{tabular}{|c|c|c|c|}
\hline Entry & $\begin{array}{c}\text { Base } \\
(10 \mathrm{~mol} \%)\end{array}$ & $\mathbf{2 a}$ (equiv.) & Yield $(\%)^{b}$ \\
\hline 1 & - & 2 & 3 \\
\hline 2 & $\mathrm{NaOH}$ & 2 & 9 \\
\hline 3 & $\mathrm{KOH}$ & 2 & 10 \\
\hline 4 & $\mathrm{KO}^{t} \mathrm{Bu}$ & 2 & 11 \\
\hline 5 & $\mathrm{~K}_{2} \mathrm{CO}_{3}$ & 2 & 11 \\
\hline 6 & $\mathrm{Na}_{2} \mathrm{CO}_{3}$ & 2 & 40 \\
\hline 7 & $\mathrm{Na}_{2} \mathrm{CO}_{3}$ & 4 & 22 \\
\hline 8 & $\mathrm{Na}_{2} \mathrm{CO}_{3}$ & 10 & 16 \\
\hline 9 & $\mathrm{NaOAc}$ & 2 & 26 \\
\hline 10 & $\mathrm{NaOAc}$ & 4 & 53 \\
\hline 11 & $\mathrm{NaOAc}$ & 10 & 61 \\
\hline
\end{tabular}

\footnotetext{
${ }^{\mathrm{a}}$ Reaction conditions: $1 \mathrm{a}(0.5 \mathrm{mmol})$, base $(0.05 \mathrm{mmol})$, solvent-free, $100{ }^{\circ} \mathrm{C}, 16 \mathrm{~h}$, under argon. ${ }^{\mathrm{b}} \mathrm{GC}$ yield based on benzonitrile, n-hexadecane used as internal standard, average of two reactions.
}

Different copper-NHC complexes ${ }^{21}$ were then tested in order to activate the nitrile (Table 2). Gratifyingly, these complexes achieved moderate to good conversion depending on the nature of the NHC ligand (Table 2, entries 1-4). Among these, $[\mathrm{Cu}(\mathrm{Cl})(\mathrm{IPr})](\mathrm{IPr}=N, N$ 'bis[(2,6-(di-iso-propyl)phenyl)]imidazol-2-ylidene) was chosen for further optimization due to its higher activity. Better conversion was achieved by either increasing the catalyst loading to 2 mol\% (Table 2 , entry 5) or using 4 equiv. of $\mathbf{2 a}$ (Table 2 , entry 6). It is worth underlining that $[\mathrm{Cu}(\mathrm{Cl})(\mathrm{IPr})]$ showed better activity compared to $\mathrm{CuCl}$, even when $10 \mathrm{~mol} \%$ of the latter was used (Table 2, entries 7 and 8). 
Considering the good conversion reached when using copper-NHC catalyst and the moderate reactivity of the system when the catalyst is a base, we were interested in exploring the synergic effect of Cu complex and base together. To investigate this possible effect, a catalytic amount of different bases (10 mol\%) was tested in the presence of [Cu(Cl)(IPr)] (1 mol\%) (Table 3). Among these, only NaOAc was found to be a suitable base (Table 3, entry 3), with a higher conversion obtained compared to the base-free test (Table 2 , entry 4). The ideal stoichiometry of $2 \mathbf{a}$ was investigated and an optimal value was found to be 2 equivalents (Table 3, entry 3). Satisfyingly, with an increase in Cu loading to 2 mol\% (Table 3 , entry 6), 95\% conversion toward za was observed. The temperature was found to be an important parameter, since any variation from $100{ }^{\circ} \mathrm{C}$ caused a significant drop in the conversion (Table 3 , entries 7 and 8).

Table 2. Screening of catalysts for the synthesis of 2-phenyl-4,5-dihydrooxazole ${ }^{a}$

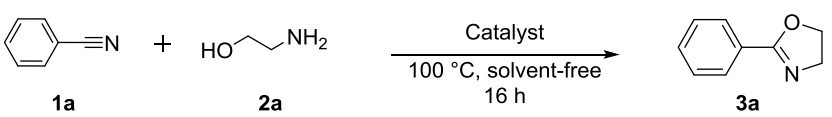

\begin{tabular}{|c|c|c|}
\hline Entry & Catalyst (mol\%) & Yield $(\%)^{b}$ \\
\hline 1 & {$[\mathrm{Cu}(\mathrm{Cl})(\mathrm{SIMes})](\mathrm{1})$} & 50 \\
\hline 2 & {$[\mathrm{Cu}(\mathrm{Cl})(\mathrm{IMes})](1)$} & 52 \\
\hline 3 & {$[\mathrm{Cu}(\mathrm{Cl})(\mathrm{SIPr})](1)$} & 67 \\
\hline 4 & {$[\mathrm{Cu}(\mathrm{Cl})(\mathrm{IPr})](1)$} & 73 \\
\hline 5 & {$[\mathrm{Cu}(\mathrm{Cl})(\mathrm{IPr})](2)$} & 83 \\
\hline $6^{\mathrm{c}}$ & {$[\mathrm{Cu}(\mathrm{Cl})(\mathrm{IPr})](1)$} & 77 \\
\hline 7 & $\mathrm{CuCl}(1)$ & 49 \\
\hline 8 & $\mathrm{CuCl}(10)$ & 65 \\
\hline
\end{tabular}

\footnotetext{
${ }^{\mathrm{a}}$ Reaction conditions: $1 \mathrm{a}(0.5 \mathrm{mmol}), \mathbf{2 a}(1 \mathrm{mmol})$, catalyst (0.005-0.05 mmol), solvent-free, $100{ }^{\circ} \mathrm{C}, 16 \mathrm{~h}$, under argon. ${ }^{\mathrm{b}} \mathrm{GC}$ yield based on benzonitrile, $n$-hexadecane used as internal standard, average of two reactions. ${ }^{c} \mathbf{2}$ mmol of $\mathbf{2 a}$.
} 
Table 3. Screening of bases for the synthesis of 2-phenyl-4,5-dihydrooxazole with $[\mathrm{Cu}(\mathrm{Cl})(\mathrm{IPr})]^{\mathrm{a}}$

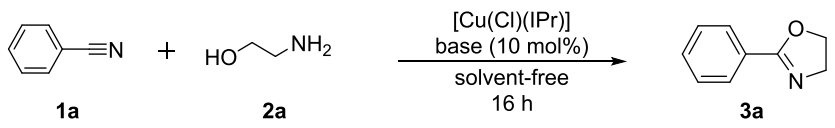

\begin{tabular}{|c|c|c|c|c|c|}
\hline Entry & Base & $\begin{array}{c}{[\mathrm{Cu}(\mathrm{Cl})(\mathrm{IPr})]} \\
(\mathrm{mol} \%)\end{array}$ & $\begin{array}{c}\mathbf{2 a} \\
\text { (equiv.) }\end{array}$ & $\begin{array}{c}\mathrm{T} \\
\left({ }^{\circ} \mathrm{C}\right)\end{array}$ & $\begin{array}{c}\text { Yield }^{\mathrm{b}} \\
(\%)\end{array}$ \\
\hline 1 & $\mathrm{~K}_{2} \mathrm{CO}_{3}$ & 1 & 2 & 100 & 11 \\
\hline 2 & $\mathrm{Na}_{2} \mathrm{CO}_{3}$ & 1 & 2 & 100 & 57 \\
\hline 3 & $\mathrm{NaOAc}$ & 1 & 2 & 100 & 90 \\
\hline 4 & $\mathrm{NaOAc}$ & 1 & 1.1 & 100 & 58 \\
\hline 5 & $\mathrm{NaOAc}$ & 1 & 4 & 100 & 88 \\
\hline 6 & NaOAc & 2 & 2 & 100 & 95 (93) \\
\hline 7 & $\mathrm{NaOAc}$ & 2 & 2 & 80 & 62 \\
\hline 8 & $\mathrm{NaOAc}$ & 2 & 2 & 120 & 88 \\
\hline
\end{tabular}

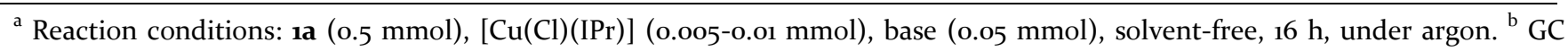
yield based on benzonitrile, $n$-hexadecane used as internal standard, average of two reactions; isolated yield in parentheses.

To highlight the superior performance of solvent-free conditions, various solvents were tested using the optimal conditions (Table 4). Among these, the reaction in methanol (Table 4, entry 5) gave a comparable yield to the reaction in the absence of solvent; however, in order to develop a less wasteful procedure, solvent-free conditions were maintained.

Table 4. Screening of solvents for the synthesis of 2-phenyl-4,5-dihydrooxazoles ${ }^{\mathrm{a}}$

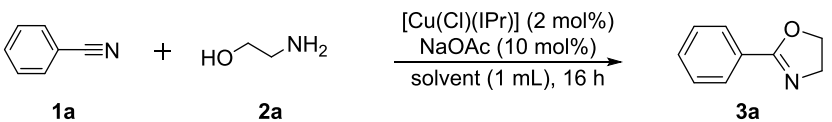




\begin{tabular}{|c|c|c|c|}
\hline Entry & Solvent & $\mathrm{T}\left({ }^{\circ} \mathrm{C}\right)$ & Yield $(\%)^{b}$ \\
\hline 1 & Toluene & 100 & 15 \\
\hline 2 & Benzene & 100 & 15 \\
\hline 3 & 1,4-Dioxane & 100 & 3 \\
\hline 4 & $\mathrm{H}_{2} \mathrm{O}$ & 100 & o \\
\hline 5 & $\mathrm{MeOH}$ & 100 & 92 \\
\hline 6 & $\mathrm{MeOH}$ & 80 & 77 \\
\hline 7 & $\mathrm{EtOH}$ & 80 & 16 \\
\hline 8 & $i \mathrm{PrOH}$ & 80 & 3 \\
\hline
\end{tabular}

${ }^{\text {a }}$ Reaction conditions: $1 \mathbf{a}(0.5 \mathrm{mmol}), \mathbf{2 a}(1 \mathrm{mmol}),[\mathrm{Cu}(\mathrm{Cl})(\mathrm{IPr})](0.005 \mathrm{mmol}), \mathrm{NaOAc}(0.05 \mathrm{mmol})$, solvent $(1 \mathrm{~mL}), 100{ }^{\circ} \mathrm{C}, 16 \mathrm{~h}$, under argon. ${ }^{\mathrm{b}} \mathrm{GC}$ yield based on benzonitrile, $n$-hexadecane used as internal standard, average of two reactions.

With these reaction conditions in hand, the scope of the reaction was investigated with a range of nitrile derivatives (Figure 1). 


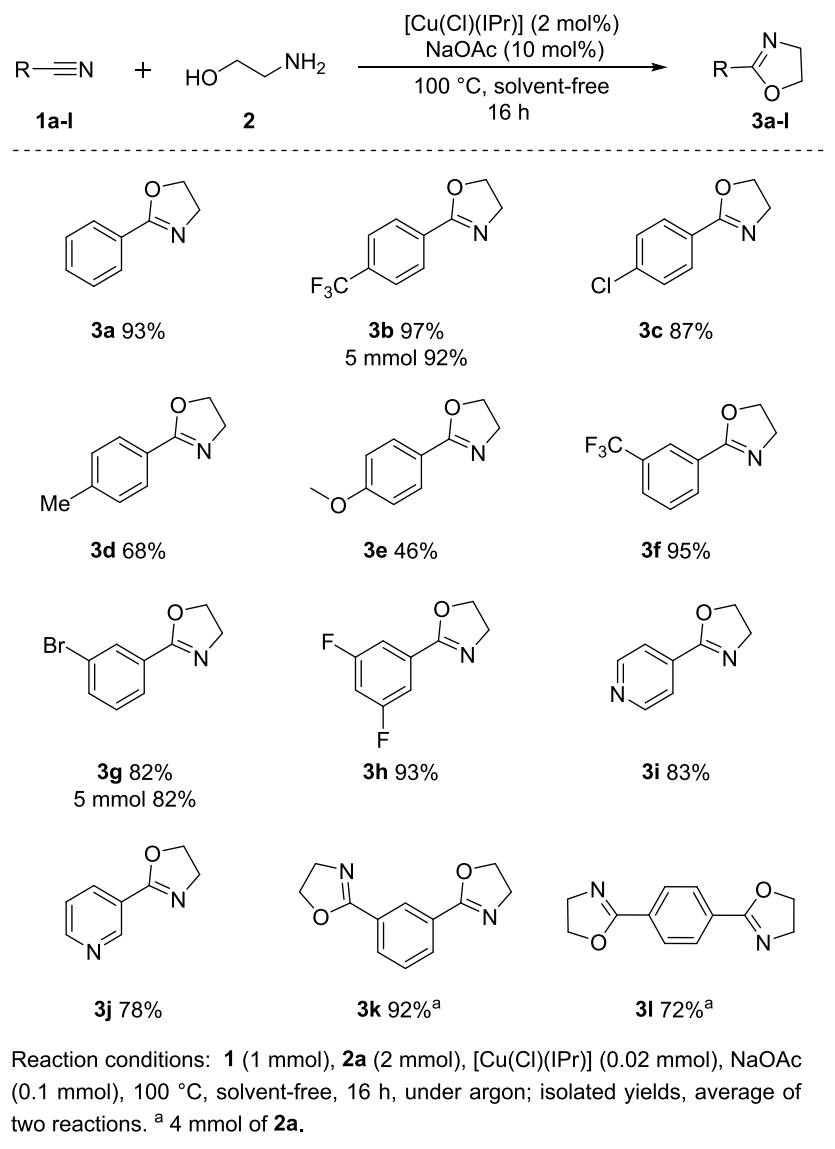

Figure 1. Formation of 2-substituted oxazolines from nitriles and ethanolamine.

Considering para-substituted benzonitriles, in which the steric effects are negligible, the outcome of the reaction depends mainly on the electronic nature of the substituents. Benzonitriles bearing electron-withdrawing groups (EWGs), such as trifluoromethyl- (3b) and chloro- (3c) substituents, gave excellent isolated yields. Moderate results were obtained when electron-donating groups (EDGs), such as methyl- (3d) and methoxy- (3e) moieties, are present. Likewise, substitution in the meta-position with EWGs gave good to excellent yields ( $\mathbf{f} \mathbf{f}-\mathbf{3 h}$ ). Ortho-substituted benzonitriles do not react under these conditions, possibly due to steric hindrance in the proximity of the nitrile moiety. Heteroaromatic nitriles were converted to the corresponding oxazolines in good yields (3i-3j). When $\mathbf{1 , 3}$-dicyano- and 1,4 -dicyanobenzene were used, the selective double functionalization was achieved by simply increasing the amount of $\mathbf{2 a}$ to 4 equivalents (3k-3l). ${ }^{22}$ When alkyl nitriles were tested, no conversion was observed. Moreover, scaling-up reactions with two selected substrates (3b and $\mathbf{3 g}$ ) to $5 \mathrm{mmol}$ gave comparable isolated yields with respect to the small scale experiments ( $1 \mathrm{mmol}$ ), allowing the synthesis of these important compounds in gram quantities. Gratifyingly, substitutions in the aliphatic chain of the aminoalcohol were well tolerated by this catalytic system (Figure 2), yielding the corresponding products in excellent yields $(3 \mathbf{m}-3 \mathbf{p})$. 


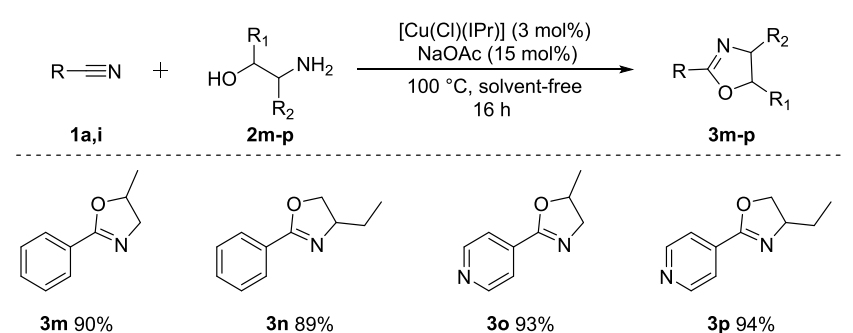

Reaction conditions: nitrile (1 mmol), $2(3 \mathrm{mmol}),[\mathrm{Cu}(\mathrm{Cl})(\mathrm{Pr})](0.03 \mathrm{mmol}), \mathrm{NaOAc}(0.15 \mathrm{mmol})$, $100{ }^{\circ} \mathrm{C}$, solvent-free, $16 \mathrm{~h}$, under argon; isolated yields, average of two reactions.

Figure 2. Formation of 2-substitued oxazolines from nitriles and aminoalcohols.

\section{CONCLUSION}

The copper-NHC complex $[\mathrm{Cu}(\mathrm{Cl})(\mathrm{IPr})]$ was found to be an active catalyst in the presence of a base co-catalyst for the conversion of different nitriles into the corresponding 2-substituted oxazolines. Gratifyingly, good to excellent isolated yields of the final products were achieved using a minimum excess of aminoalcohols and only a substoichiometric amount of base (1o mol\% of $\mathrm{NaOAc}$ ) under solvent-free conditions.

\section{EXPERIMENTAL SECTION}

General information. ${ }^{1} \mathrm{H},{ }^{13} \mathrm{C}\left\{{ }^{1} \mathrm{H}\right\}$ and ${ }^{19} \mathrm{~F}\left\{{ }^{1} \mathrm{H}\right\}$ Nuclear Magnetic Resonance (NMR) spectra were recorded at 298K using the residual solvent peak for ${ }^{13} \mathrm{C}\left\{{ }^{1} \mathrm{H}\right\}$ NMR $\left(\mathrm{CDCl}_{3}: \delta \mathrm{C}=77.36 \mathrm{ppm}\right)$ and TMS as reference for ${ }^{1} \mathrm{H}$ NMR. Gas chromatography (GC) analyses were performed on an apparatus equipped with a flame ionization detector and a (5\%-phenyl)methylpolysiloxane column ( $30 \mathrm{~m}, 320 \mu \mathrm{m}$, film: $0.25 \mu \mathrm{m}$ ). The reaction's efficiency did not seem to be affected when carried out outside the glovebox, using Schlenk techniques, under air-free conditions; however, all reactions reported here have been conducted in a glovebox for more convenience.

Materials. All commercially available reagents were used without further purification. Analytical thin layer chromatography was performed on $0.20 \mathrm{~mm}$ silica gel plates. Flash chromatography was performed using silica gel (200-300 mesh).

General procedure for the synthesis of 2-substituted oxazolines with ethanolamine. In a glovebox, a vial was charged with $[\mathrm{Cu}(\mathrm{Cl})(\mathrm{IPr})](0.02 \mathrm{mmol}), \mathrm{NaOAc}(0.1 \mathrm{mmol})$, the nitrile $(1 \mathrm{mmol})$ and ethanolamine $(2 \mathrm{mmol})$. The reaction was stirred at $100{ }^{\circ} \mathrm{C}$ for $16 \mathrm{~h}$ under solvent-free conditions. The conversion was determined by GC analysis or by ${ }^{1} \mathrm{H}$ NMR. The crude mixture was purified by column chromatography to give the desired compound.

2-Phenyl-4,5-dihydrooxazole (3a). ${ }^{14}$ The general procedure yielded after flash chromatography $\left(\mathrm{SiO}_{2}, \mathrm{CH}_{2} \mathrm{Cl}_{2} /\right.$ ethyl acetate, 1:1) the title compound as a colorless oil (136.9 mg, 93\%). The general procedure repeated using standard Schlenk techniques yielded after flash chromatography $\left(\mathrm{SiO}_{2}, \mathrm{CH}_{2} \mathrm{Cl}_{2} /\right.$ ethyl acetate, 1:1) the title compound as a colorless oil (135.4 
mg, 92\%). ${ }^{1} \mathrm{H} \mathrm{NMR}\left(\mathrm{CDCl}_{3}, 400 \mathrm{MHz}\right): \delta$ 7.98-7.95 (m, $\left.2 \mathrm{H}\right), 7.51-7.40(\mathrm{~m}, 3 \mathrm{H}), 4.44(\mathrm{t}, 2 \mathrm{H}, J=9.8 \mathrm{~Hz}), 4.07(\mathrm{t}, 2 \mathrm{H}, J=9.8$ Hz). $\left.{ }^{13} \mathrm{C}^{1} \mathrm{H}\right\} \mathrm{NMR}\left(\mathrm{CDCl}_{3}, 100 \mathrm{MHz}\right): \delta 164.7,131.3,128.3,128.1,127.8,67.6,54.9$.

2-(4-Trifluoromethylphenyl)-4,5-dihydrooxazole (3b). The general procedure yielded after flash chromatography $\left(\mathrm{SiO}_{2}, \mathrm{CH}_{2} \mathrm{Cl}_{2}\right.$, ethyl acetate, 8:2) the title compound as a colorless solid (208.6 mg, 97\%). m.p. 104-106 ${ }^{\circ} \mathrm{C} .{ }^{1} \mathrm{H}$ NMR $\left(\mathrm{CDCl}_{3}\right.$, $400 \mathrm{MHz}): \delta 8.09(\mathrm{~d}, 2 \mathrm{H}, J=8.2 \mathrm{~Hz}), 7.7 \mathrm{O}(\mathrm{d}, 2 \mathrm{H}, J=8.2 \mathrm{~Hz}), 4.5 \mathrm{O}(\mathrm{t}, 2 \mathrm{H}, J=9.4 \mathrm{~Hz}), 4.12(\mathrm{t}, 2 \mathrm{H}, J=9.4 \mathrm{~Hz}) .{ }^{13} \mathrm{C}\left\{{ }^{1} \mathrm{H}\right\} \mathrm{NMR}$ $\left(\mathrm{CDCl}_{3}, 100 \mathrm{MHz}\right): \delta 163.4,132.8\left(\mathrm{q}, J_{\mathrm{C}-\mathrm{F}}=33.2 \mathrm{~Hz}\right), 131.1,128.5,125.3\left(\mathrm{q}, J_{\mathrm{C}-\mathrm{F}}=3.6 \mathrm{~Hz}\right), 123.8\left(\mathrm{q}, J_{\mathrm{C}-\mathrm{F}}=272.4 \mathrm{~Hz}\right), 67.9,55.1$. ${ }^{19} \mathrm{~F}\left\{{ }^{1} \mathrm{H}\right\}$ NMR $\left(\mathrm{CDCl}_{3}, 376 \mathrm{MHz}\right): \delta$-62.19. HRMS (ESI) m/z: $[\mathrm{M}+\mathrm{H}]^{+}$Calcd for $\mathrm{C}_{10} \mathrm{H}_{8} \mathrm{~F}_{3} \mathrm{NO}$ 216.0631; Found 216.0632.

2-(4-Chlorophenyl)-4,5-dihydrooxazole (3c). ${ }^{14}$ The general procedure yielded after flash chromatography $\left(\mathrm{SiO}_{2}\right.$, hexane, ethyl acetate, $2: 8)$ the title compound as a colorless solid (157.5 mg, 87\%). m.p. $77-79{ }^{\circ} \mathrm{C} .{ }^{1} \mathrm{H}$ NMR $\left(\mathrm{CDCl}_{3}, 400 \mathrm{MHz}\right)$ : $\delta$ 7.90-7.86 (m, 2H), 7.40-7.37 (m, 2H), $4.44(\mathrm{t}, 2 \mathrm{H}, J=9.4 \mathrm{~Hz}), 4.06(\mathrm{t}, 2 \mathrm{H}, J=9.4 \mathrm{~Hz}) .{ }^{13} \mathrm{C}\left\{{ }^{1} \mathrm{H}\right\} \mathrm{NMR}\left(\mathrm{CDCl}_{3}, 100 \mathrm{MHz}\right): \delta$ $163.9,137.6,129.6,128.8,126.4,67.9,55.1$.

2-p-Tolyl-4,5-dihydrooxazole (3d). ${ }^{14}$ The general procedure yielded after flash chromatography $\left(\mathrm{SiO}_{2}\right.$, hexane, ethyl acetate, $3: 7)$ the title compound as a colorless solid (109.5 mg, 68\%). m.p. 69-71 ${ }^{\circ} \mathrm{C} .{ }^{1} \mathrm{H}$ NMR $\left(\mathrm{CDCl}_{3}, 400 \mathrm{MHz}\right): \delta 7.86-7.83$ (m, 2H), 7.24-7.21 (m, 2H), $4.42(\mathrm{t}, 2 \mathrm{H}, J=9.4 \mathrm{~Hz}), 4.05(\mathrm{t}, 2 \mathrm{H}, J=9.4 \mathrm{~Hz}) .{ }^{13} \mathrm{C}\left[{ }^{1} \mathrm{H}\right\} \mathrm{NMR}\left(\mathrm{CDCl}_{3}, 100 \mathrm{MHz}\right): \delta 164.7,141.6$ 129.0, 128.1, 125.5, 67.5, 54.9, 21.6.

2-(4-Methoxyphenyl)-4,5-dihydrooxazole (3e). ${ }^{6 a}$ The general procedure yielded after flash chromatography $\left(\mathrm{SiO}_{2}\right.$, hexane, ethyl acetate, 1:9) the title compound as a colorless solid (81.5 mg, $46 \%)$. m.p. $60-62{ }^{\circ} \mathrm{C} .{ }^{1} \mathrm{H}$ NMR $\left(\mathrm{CDCl}_{3}, 400\right.$ MHz): $\delta$ 7.89-7.85 (m, 2H), 6.91-6.87 (m, 2H), $4.37(\mathrm{t}, 2 \mathrm{H}, J=9.4 \mathrm{~Hz}), 4.00(\mathrm{t}, 2 \mathrm{H}, J=9.4 \mathrm{~Hz}), 3.81\left(\mathrm{~s},{ }_{3} \mathrm{H}\right) .{ }^{13} \mathrm{C}\left\{{ }^{1} \mathrm{H}\right\} \mathrm{NMR}$ $\left(\mathrm{CDCl}_{3}, 100 \mathrm{MHz}\right): \delta$ 164.4, 162.0, 129.9, 120.3, 113.7, 67.5, 55.3, 54.9.

2-(3-Trifluoromethylphenyl)-4,5-dihydrooxazole (3f). The general procedure yielded after flash chromatography $\left(\mathrm{SiO}_{2}, \mathrm{CH}_{2} \mathrm{Cl}_{2}\right.$, ethyl acetate, 8:2) the title compound as a colorless oil (204.3 mg, 95\%). ${ }^{1} \mathrm{H} \mathrm{NMR}\left(\mathrm{CDCl}_{3}, 400 \mathrm{MHz}\right): \delta 8.25$ $(\mathrm{s}, 1 \mathrm{H}), 8.15(\mathrm{~d}, 1 \mathrm{H}, J=7.7 \mathrm{~Hz}), 7.75(\mathrm{~d}, 1 \mathrm{H}, J=7.7 \mathrm{~Hz}), 7.57(\mathrm{t}, 1 \mathrm{H}, J=7.7 \mathrm{~Hz}), 4.50(\mathrm{t}, 2 \mathrm{H}, J=9.5 \mathrm{~Hz}), 4.12(\mathrm{t}, 2 \mathrm{H}, J=9.5 \mathrm{~Hz})$. ${ }^{13} \mathrm{C}\left\{{ }^{1} \mathrm{H}\right\} \operatorname{NMR}\left(\mathrm{CDCl}_{3}, 100 \mathrm{MHz}\right): \delta 163.4,131.9,130.9\left(\mathrm{q}, J_{\mathrm{C}-\mathrm{F}}=32.9 \mathrm{~Hz}\right), 128.9,128.6,127.7\left(\mathrm{q}, J_{\mathrm{C}-\mathrm{F}}=3.4 \mathrm{~Hz}\right), 125.1\left(\mathrm{q}, J_{\mathrm{C}-\mathrm{F}}=3.7\right.$ $\mathrm{Hz}), 123.8\left(\mathrm{q}, J_{\mathrm{C}-\mathrm{F}}=272.4 \mathrm{~Hz}\right), 67.9$, 55.0. ${ }^{19} \mathrm{~F}\left\{{ }^{1} \mathrm{H}\right\} \operatorname{NMR}\left(\mathrm{CDCl}_{3}, 376 \mathrm{MHz}\right): \delta-62.83 . \mathrm{HRMS}(\mathrm{ESI}) \mathrm{m} / \mathrm{z}:[\mathrm{M}+\mathrm{H}]^{+}$Calcd for $\mathrm{C}_{10} \mathrm{H}_{8} \mathrm{~F}_{3} \mathrm{NO} 216.0631$; Found 216.0632.

2-(3-Bromophenyl)-4,5-dihydrooxazole (3g). .e $^{\mathrm{e}}$ The general procedure yielded after flash chromatography $\left(\mathrm{SiO}_{2}\right.$, hexane, ethyl acetate, $1: 1)$ the title compound as a colorless oil $(184.5 \mathrm{mg}, 82 \%) .{ }^{1} \mathrm{H}$ NMR $\left(\mathrm{CDCl}_{3}, 400 \mathrm{MHz}\right): \delta 8.10(\mathrm{t}, 1 \mathrm{H}, J=1.8$ 
$\mathrm{Hz}), 7.87(\mathrm{dt}, 1 \mathrm{H}, J=8.0 \mathrm{~Hz}, J=1.3), 7.59(\mathrm{dq}, 1 \mathrm{H}, J=8.0 \mathrm{~Hz}, J=1.3), 7.27(\mathrm{t}, 1 \mathrm{H}, J=8.0 \mathrm{~Hz}), 4.43(\mathrm{t}, 2 \mathrm{H}, J=9.6 \mathrm{~Hz}), 4.06(\mathrm{t}$, $2 \mathrm{H}, J=9.6 \mathrm{~Hz}) \cdot{ }^{13} \mathrm{C}\left\{{ }^{1} \mathrm{H}\right\} \mathrm{NMR}\left(\mathrm{CDCl}_{3}, 100 \mathrm{MHz}\right): \delta 163.4,134 \cdot 3,131.2,130.0,129.8,126.8,122.4,67.9,55.0$.

2-(3,5-Difluorophenyl)-4,5-dihydrooxazole (3h). The general procedure yielded after flash chromatography $\left(\mathrm{SiO}_{2}\right.$, hexane, ethyl acetate, 6:4) the title compound as a colorless solid (170.2 mg, 93\%). m.p. $74-76{ }^{\circ} \mathrm{C} .{ }^{1} \mathrm{H}$ NMR $\left(\mathrm{CDCl}{ }_{3}, 400\right.$ $\mathrm{MHz}): \delta 7.50-7.44(\mathrm{~m}, 2 \mathrm{H}), 6.92\left(\mathrm{tt},{ }_{1} \mathrm{H}, J=8.8 \mathrm{~Hz}, 2.4 \mathrm{~Hz}\right), 4.45(\mathrm{t}, 2 \mathrm{H}, J=9.4 \mathrm{~Hz}), 4.08(\mathrm{t}, 2 \mathrm{H}, J=9.4 \mathrm{~Hz}) .{ }^{13} \mathrm{C}\left\{{ }^{1} \mathrm{H}\right\} \mathrm{NMR}$ $\left(\mathrm{CDCl}_{3}, 100 \mathrm{MHz}\right): \delta 162.7,162.6\left(\mathrm{dd}, J_{\mathrm{C}-\mathrm{F}}=235.7 \mathrm{~Hz}, J_{\mathrm{C}-\mathrm{F}}=12.5 \mathrm{~Hz}\right), 130.9\left(\mathrm{t}, J_{\mathrm{C}-\mathrm{F}}=10.4 \mathrm{~Hz}\right), 111.3\left(\mathrm{dd}, J_{\mathrm{C}-\mathrm{F}}=19.6 \mathrm{~Hz}, J_{\mathrm{C}-\mathrm{F}}=7.8\right.$ $\mathrm{Hz}), 106.6\left(\mathrm{t}, J_{\mathrm{C}-\mathrm{F}}=25.5 \mathrm{~Hz}\right), 68.0,55.0 .{ }^{19} \mathrm{~F}\left\{{ }^{1} \mathrm{H}\right\} \mathrm{NMR}\left(\mathrm{CDCl}_{3}, 376 \mathrm{MHz}\right): \delta-109.05 . \mathrm{HRMS}(\mathrm{ESI}) \mathrm{m} / \mathrm{z}:[\mathrm{M}+\mathrm{H}]^{+} \mathrm{Calcd}$ for $\mathrm{C}_{9} \mathrm{H}_{8} \mathrm{~F}_{2} \mathrm{NO}$ 184.0568; Found 184.0565.

2-(Pyridin-4-yl)-4,5-dihydrooxazole (3i). ${ }^{14}$ The general procedure yielded after flash chromatography $\left(\mathrm{SiO}_{2}\right.$, ethyl acetate) the title compound as a white solid (122.9 mg, 83\%). m.p. 109-111 ${ }^{\circ} \mathrm{C} .{ }^{1} \mathrm{H} \mathrm{NMR}\left(\mathrm{CDCl}_{3}, 400 \mathrm{MHz}\right): \delta 8.72(\mathrm{~s}, 2 \mathrm{H}), 7.78$ $(\mathrm{s}, 2 \mathrm{H}), 4.47(\mathrm{t}, 2 \mathrm{H}, J=9.7 \mathrm{~Hz}), 4.10(\mathrm{t}, 2 \mathrm{H}, J=9.7 \mathrm{~Hz}) \cdot{ }^{13} \mathrm{C}\left\{{ }^{1} \mathrm{H}\right\} \mathrm{NMR}\left(\mathrm{CDCl}_{3}, 100 \mathrm{MHz}\right): \delta$ 163.0, 150.3, 135.0, 122.0, 68.0, 55.1.

2-(Pyridin-3-yl)-4,5-dihydrooxazole (3j). ${ }^{14}$ The general procedure yielded after flash chromatography $\left(\mathrm{SiO}_{2}\right.$, ethyl acetate) the title compound as a white solid (115.5 mg, 78\%). m.p. 68-70 ${ }^{\circ} \mathrm{C} .{ }^{1} \mathrm{H} \mathrm{NMR}\left(\mathrm{CDCl}_{3}, 400 \mathrm{MHz}\right): \delta 9.15(\mathrm{~s}, 2 \mathrm{H}), 8.71^{-}$ $8.69(\mathrm{~m}, 1 \mathrm{H}), 8.21(\mathrm{dt}, 1 \mathrm{H}, J=8.0 \mathrm{~Hz}, J=1.9 \mathrm{~Hz}), 7 \cdot 36-7.34(\mathrm{~m}, 1 \mathrm{H}), 4.46(\mathrm{t}, 2 \mathrm{H}, J=9.7 \mathrm{~Hz}), 4.08(\mathrm{t}, 2 \mathrm{H}, J=9.7 \mathrm{~Hz}) .{ }^{13} \mathrm{C}\left\{{ }^{1} \mathrm{H}\right\}$ $\operatorname{NMR}\left(\mathrm{CDCl}_{3}, 100 \mathrm{MHz}\right): \delta 162.7,152.0,149.4,135 \cdot 5,123.9,123.2,67.8,55.0$.

1,3-Bis $\left(4,5\right.$-dihydrooxazol-2-yl)benzene $(3 \mathbf{k}) .{ }^{14}$ The general procedure yielded after flash chromatography $\left(\mathrm{SiO}_{2}\right.$, $\mathrm{CH}_{2} \mathrm{Cl}_{2}$, ethyl acetate, 1:1) the title compound as a white solid (198.8 mg, 92\%). m.p. 137-139 ${ }^{\circ} \mathrm{C} .{ }^{1} \mathrm{H}^{\mathrm{NMR}}\left(\mathrm{CDCl}{ }_{3}, 400 \mathrm{MHz}\right)$ : $\delta 8.49-8.48(\mathrm{~m}, \mathrm{1H}), 8.07(\mathrm{dd}, 2 \mathrm{H}, J=7.8 \mathrm{~Hz}, 1.7 \mathrm{~Hz}), 8.07(\mathrm{t}, 1 \mathrm{H}, J=7.8 \mathrm{~Hz}), 4.44\left(\mathrm{t},{ }_{4} \mathrm{H}, J=9.4 \mathrm{~Hz}\right), 4.07(\mathrm{t}, 4 \mathrm{H}, J=9.4$ $\mathrm{Hz}) .{ }^{13} \mathrm{C}\left\{{ }^{1} \mathrm{H}\right\} \mathrm{NMR}\left(\mathrm{CDCl}_{3}, 100 \mathrm{MHz}\right): \delta 164.1,130.9,128.5,128.2,128.0,67.8,55.1$.

1,4-Bis $\left(4,5\right.$-dihydrooxazol-2-yl)benzene $\left(3^{1}\right){ }^{14}$ The general procedure yielded after flash chromatography $\left(\mathrm{SiO}_{2}\right.$, $\mathrm{CH}_{2} \mathrm{Cl}_{2}$, ethyl acetate, 1:1) the title compound as a white solid (156.3 mg, 72\%). m.p. $238-240{ }^{\circ} \mathrm{C} .{ }^{1} \mathrm{H}$ NMR $\left(\mathrm{CDCl}_{3}\right.$, 400 $\mathrm{MHz}): \delta 7.99(\mathrm{~s}, 4 \mathrm{H}), 4.45(\mathrm{t}, 4 \mathrm{H}, J=9.6 \mathrm{~Hz}), 4.08(\mathrm{t}, 4 \mathrm{H}, J=9.6 \mathrm{~Hz}) .{ }^{13} \mathrm{C}\left\{{ }^{1} \mathrm{H}\right\} \mathrm{NMR}(\mathrm{CDCl}, 100 \mathrm{MHz}): \delta 164.2,130.3,128.2$ $67.8,55.1$.

General procedure for the synthesis of 2-substituted oxazolines with substituted aminoalcohols. In a glovebox, a vial was charged with $[\mathrm{Cu}(\mathrm{Cl})(\mathrm{IPr})](0.03 \mathrm{mmol})$, NaOAc (o.15 mmol), the nitrile $(1 \mathrm{mmol})$ and aminoalcohol (3 mmol). The reaction was stirred at $100{ }^{\circ} \mathrm{C}$ for $16 \mathrm{~h}$ under solvent-free conditions. The conversion was determined by ${ }^{1} \mathrm{H}$ NMR. The crude mixture was purified by column chromatography to give the desired compound. 
5-Methyl-2-phenyl-4,5-dihydrooxazole (3m). ${ }^{6 c}$ The general procedure yielded after flash chromatography $\left(\mathrm{SiO}_{2}\right.$, $\mathrm{CH}_{2} \mathrm{Cl}_{2}$, ethyl acetate, 8:2) the title compound as a colorless oil (145 mg, 90\%). ${ }^{1} \mathrm{H}$ NMR $\left(\mathrm{CDCl}_{3}, 400 \mathrm{MHz}\right): \delta 7.96(\mathrm{~d}, 2 \mathrm{H}, J$ $=7.4 \mathrm{~Hz}), 7.51-7.47(\mathrm{~m}, 1 \mathrm{H}), 7.42(\mathrm{t}, 2 \mathrm{H}, J=7.4 \mathrm{~Hz}), 4.90-4.83(\mathrm{~m}, 1 \mathrm{H}), 4.19-4.14(\mathrm{~m}, 1 \mathrm{H}), 3.63(\mathrm{q}, 1 \mathrm{H}, J=6.2 \mathrm{~Hz}), 1.45\left(\mathrm{~d},{ }_{3} \mathrm{H}\right.$, $J=6.2 \mathrm{~Hz}) .{ }^{13} \mathrm{C}\left\{{ }^{1} \mathrm{H}\right\} \operatorname{NMR}\left(\mathrm{CDCl}_{3}, 100 \mathrm{MHz}\right): \delta$ 163.9, 131.2, 128.3, 128.1, 76.3, 61.6, 21.2. 1 Cq missing.

4-Ethyl-2-phenyl-4,5-dihydrooxazole (3n). ${ }^{23}$ The general procedure yielded after flash chromatography $\left(\mathrm{SiO}_{2}, \mathrm{CH}_{2} \mathrm{Cl}_{2}\right.$, ethyl acetate, 8:2) the title compound as a colorless oil (155.8 mg, 89\%). ${ }^{1} \mathrm{H}$ NMR $\left(\mathrm{CDCl}_{3}, 400 \mathrm{MHz}\right): \delta$ 7.99-7.96 (m, $\left.2 \mathrm{H}\right)$, 7.51-7.47 (m, $1 \mathrm{H}), 7 \cdot 45-7.40(\mathrm{~m}, 2 \mathrm{H}), 4.52-4.48(\mathrm{~m}, 1 \mathrm{H}), 4.31-4.23(\mathrm{~m}, 1 \mathrm{H}), 4.08(\mathrm{t}, 1 \mathrm{H}, J=7.9 \mathrm{~Hz}), 1.85^{-1.75}(\mathrm{~m}, 1 \mathrm{H}), 1.69-1.58$ $(\mathrm{m}, 1 \mathrm{H}), 1.02(\mathrm{t}, 3 \mathrm{H}, J=7.5 \mathrm{~Hz}) .{ }^{13} \mathrm{C}\left\{{ }^{1} \mathrm{H}\right\} \mathrm{NMR}\left(\mathrm{CDCl}_{3}, 100 \mathrm{MHz}\right): \delta$ 163.4, 131.2, 127.9, 72.1, 68.0, 28.6, 10.o.

5-Methyl-2-(pyridin-4-yl)-4,5-dihydrooxazole (30). The general procedure yielded after flash chromatography $\left(\mathrm{SiO}_{2}\right.$, ethyl acetate) the title compound as a colorless oil (150.7 mg, 93\%). ${ }^{1} \mathrm{H}$ NMR $\left(\mathrm{CDCl}_{3}, 400 \mathrm{MHz}\right): \delta 8.74(\mathrm{~s}, 2 \mathrm{H}), 7.79-7.78$ $(\mathrm{m}, 2 \mathrm{H}), 4.94-4.85(\mathrm{~m}, 1 \mathrm{H}), 4.21-4.15(\mathrm{~m}, 1 \mathrm{H}), 3.65(\mathrm{q}, 1 \mathrm{H}, J=6.2 \mathrm{~Hz}), 1.44(\mathrm{~d}, 3 \mathrm{H}, J=6.2 \mathrm{~Hz}) .{ }^{13} \mathrm{C}\left\{{ }^{1} \mathrm{H}\right\} \mathrm{NMR}\left(\mathrm{CDCl}_{3}, 100\right.$ MHz): $\delta$ 162.2, 150.2, 135.3, 122.0, 76.9, 61.7, 21.1. HRMS (ESI) m/z: [M + H] $]^{+}$Calcd for $\mathrm{C}_{9} \mathrm{H}_{11} \mathrm{O}_{1} \mathrm{~N}_{2}$ 163.0866; Found 163.0862.

4-Ethyl-2-(pyridin-4-yl)-4,5-dihydrooxazole (3p). The general procedure yielded after flash chromatography $\left(\mathrm{SiO}_{2}\right.$, ethyl acetate) the title compound as a colorless oil (165.5 mg, 94\%). ${ }^{1} \mathrm{H} \mathrm{NMR}\left(\mathrm{CDCl}_{3}, 400 \mathrm{MHz}\right): \delta 8.73(\mathrm{~s}, 2 \mathrm{H}), 7.79(\mathrm{~s}, 2 \mathrm{H})$, 4.54-4.48 (m, $1 \mathrm{H})$, 4.32-4.23 (m, $1 \mathrm{H})$, 4.11-4.06 (m, $1 \mathrm{H}), 1.82-1.72(\mathrm{~m}, 1 \mathrm{H})$, 1.68-1.57 (m, $1 \mathrm{H})$, 1.02-0.98 (m, $\left.{ }_{3} \mathrm{H}\right) .{ }^{13} \mathrm{C}\left\{{ }^{1} \mathrm{H}\right\} \mathrm{NMR}$ $\left(\mathrm{CDCl}_{3}, 100 \mathrm{MHz}\right): \delta 161.8,150.2,135.2,122.1,72.5,68.2,28.5$, 10.o. HRMS (ESI) m/z: $[\mathrm{M}+\mathrm{H}]^{+}$Calcd for $\mathrm{C}_{10} \mathrm{H}_{13} \mathrm{O}_{1} \mathrm{~N}_{2}$ 177.1022; Found 177.1020.

\section{General procedure for the synthesis of 2-substituted oxazolines - $5 \mathrm{mmol}$ scale}

In the glovebox, a flame dried schlenck was charged with $[\mathrm{Cu}(\mathrm{Cl})(\mathrm{IPr})](\mathrm{o} .1 \mathrm{mmol})$, $\mathrm{NaOAc}$ (o.5 mmol), the nitrile $(5$ mmol) and ethanolamine (10 mmol). The reaction was stirred at $100{ }^{\circ} \mathrm{C}$ for $16 \mathrm{~h}$ under solvent-free conditions. The conversion was determined by GC analysis or by ${ }^{1} \mathrm{H}$ NMR. The crude mixture was purified by column chromatography to give the desired compound.

2-(4-Trifluoromethylphenyl)-4,5-dihydrooxazole (3b). The general procedure yielded after flash chromatography $\left(\mathrm{SiO}_{2}, \mathrm{CH}_{2} \mathrm{Cl}_{2}\right.$, ethyl acetate, 8:2) the title compound as a colorless solid (990 mg, 92\%). m.p. 104-106 ${ }^{\circ} \mathrm{C} .{ }^{1} \mathrm{H}$ NMR $\left(\mathrm{CDCl}_{3}\right.$, $400 \mathrm{MHz}): \delta 8.09(\mathrm{~d}, 2 \mathrm{H}, J=8.2 \mathrm{~Hz}), 7.70(\mathrm{~d}, 2 \mathrm{H}, J=8.2 \mathrm{~Hz}), 4.50(\mathrm{t}, 2 \mathrm{H}, J=9.4 \mathrm{~Hz}), 4.12(\mathrm{t}, 2 \mathrm{H}, J=9.4 \mathrm{~Hz}) .{ }^{13} \mathrm{C}\left\{{ }^{1} \mathrm{H}\right\} \mathrm{NMR}$ $\left(\mathrm{CDCl}_{3}, 100 \mathrm{MHz}\right): \delta 163.4,132.8\left(\mathrm{q}, J_{\mathrm{C}-\mathrm{F}}=33.2 \mathrm{~Hz}\right), 131.1,128.5,125.3\left(\mathrm{q}, J_{\mathrm{C}-\mathrm{F}}=3.6 \mathrm{~Hz}\right), 123.8\left(\mathrm{q}, J_{\mathrm{C}-\mathrm{F}}=272.4 \mathrm{~Hz}\right), 67.9,55.1$. ${ }^{19} \mathrm{~F}\left\{{ }^{1} \mathrm{H}\right\}$ NMR $\left(\mathrm{CDCl}_{3}, 376 \mathrm{MHz}\right): \delta$-62.19. HRMS (ESI) m/z: $[\mathrm{M}+\mathrm{H}]^{+}$Calcd for $\mathrm{C}_{10} \mathrm{H}_{8} \mathrm{~F}_{3} \mathrm{NO} 216.0631$; Found 216.0632. 
2-(3-Bromophenyl)-4,5-dihydrooxazole $(3 \mathrm{~g}) .^{3}$ The general procedure yielded after flash chromatography $\left(\mathrm{SiO}_{2}\right.$, hexane, ethyl acetate, 1:1) the title compound as a colorless oil (927 mg, 82\%). ${ }^{1} \mathrm{H}$ NMR $\left(\mathrm{CDCl}_{3}, 400 \mathrm{MHz}\right): \delta 8.10(\mathrm{t}, 1 \mathrm{H}, J=1.8$ $\mathrm{Hz}), 7.87(\mathrm{dt}, 1 \mathrm{H}, J=8.0 \mathrm{~Hz}, J=1.3), 7.59(\mathrm{dq}, 1 \mathrm{H}, J=8.0 \mathrm{~Hz}, J=1.3), 7.27(\mathrm{t}, 1 \mathrm{H}, J=8.0 \mathrm{~Hz}), 4.43(\mathrm{t}, 2 \mathrm{H}, J=9.6 \mathrm{~Hz}), 4.06(\mathrm{t}$, $2 \mathrm{H}, J=9.6 \mathrm{~Hz}) .{ }^{13} \mathrm{C}\left\{{ }^{1} \mathrm{H}\right\} \mathrm{NMR}\left(\mathrm{CDCl}_{3}, 100 \mathrm{MHz}\right): \delta 163.4,134.3,131.2,130.0,129.8,126.8,122.4,67.9,55.0$.

\section{ASSOCIATED CONTENT}

Supporting Information. Optimization of reaction conditions, ${ }^{1} \mathrm{H}$ and ${ }^{13} \mathrm{C}\left\{{ }^{1} \mathrm{H}\right\}$ NMR spectra of $3 \mathrm{a}-3 \mathrm{p}$. This material is available free of charge via the Internet at http://pubs.acs.org.

\section{AUTHOR INFORMATION}

\section{Corresponding Author}

*E-mail: cc111@st-andrews.ac.uk; Fax: +44 (o)1334463808

\section{ACKNOWLEDGMENT}

The authors gratefully acknowledge the Royal Society (University research Fellowship to C.S.J.C) for funding. We also thank the EPSRC UK National Mass Spectrometry Facility at Swansea University.

\section{REFERENCES}

(1) (a) Tsuda, M.; Yamakawa, M.; Oka, S.; Tanaka, Y.; Hoshino, Y.; Mikami, Y.; Sato, A.; Fujiwara, H.; Ohizumi, Y.; Kobayashi, J. J. Nat. Prod. 2005, 68, 462. (b) Onishi, H. R.; Pelak, B. A.; Silver, L. L.; Kahan, F. M.; Chen, M.-H.; Patchett, A. A.; Galloway, S. M.; Hyland, S. A.; Anderson, M. S.; Raetz, C. R. H. Science 1996, 274, 980. (c) Li, Q.; Woods, K. W.; Claireborne, A.; Gwanltey, S. L., II; Barr, K. J.; Liu, G.; Gehrke, L.; Credo, R. B.; Hua Hui, Y.; Lee, J.; Warner, R. B.; Kovar, P.; Nukkala, M. A.; Zielinski, N. A.; Tahir, S. K.; Fitzgerald, M.; Kim, K. H.; Marsh, K.; Frost, D.; Ng, S. -C.; Rosenberg, S.; Sham, H. L. Bioorg. Med. Chem. 2oo2, 12, 465. (d) Tsukamoto, M.; Murooka, K.; Nakajima, S.; Abe, S.; Suzuki, H.; Hirano, K.; Kondo, H.; Kojiri, K.; Suda, H. J. Antibiot. 1997, 5o, 815. (e) Vizi, E. S. Med. Res. Rev. 1986, 6, 431. (f) Bergeron, R. J.; Xin, M. G.; Weimar, W. R.; Smith, R. E.; Wiegand, J. J. Med. Chem. 2oo1, 44, 2469.

(2) (a) Ghosh, A. K.; Mathivanan, P.; Cappiello, J. Tetrahedron Asymmetry 1998, 9, 1. (b) Hargaden, G. C.; Guiry, P. J. Chem. Rev. 20o9, 109, 2505 .

(3) (a) Frump, J. A. Chem. Rev. 1971, 71, 483. (b) Reuman, M.; Meyers, A. I. Tetrahedron 1985, 41, 837. (c) Gant, T. G.; Meyers, A. I. Tetrahedron 1994, 50, 2297.

(4) (a) Saravanan, P.; Corey, E. J. J. Org. Chem. 2003, 68, 276o. (b) Lee, Y. -J.; Lee, J.; Kim, M. -J.; Jeong, B. -S.; Lee, J. -H.; Kim, T. -S.; Lee, J.; Ku, J. -M.; Jew, S. -S.; Park, H. -G. Org. Lett. 2005, 7, 3207. 
(5) For selected examples, see (a) Wu, X. -F.; Neumann, H.; Neumann, S.; Beller, M. Chem. Eur. J. 2012, 18, 13619. (b) Boissarie, P. J.; Hamilton, Z. E.; Lang, S.; Murphy, J. A.; Suckling, C. J. Org. Lett. 2o11, 13, 6256. (c) Doherty, S.; Knight, J. G.; Hashmi, A. S. K.; Smyth, C. H.; Ward, N. A. B.; Robson, K. J.; Tweedley, S.; Harrington, R. W.; Clegg, W. Organometallics 2o1o, 29, 4139. (d) Maekawa, K.; Sasaki, T.; Kubo, K.; Igarashia, T.; Sakurai, T. Tetrahedron Lett. 2004, 45, 3663. (e) Garg, P.; Chaudhary, S.; Milton, M. D. J. Org. Chem. 2014, 79,8668 .

(6) For selected examples about the cyclodehydration of $\beta$-hydroxy amides, see (a) Xu, Q.; Z. Li, Z. Tetrahedron Lett. $2009,50,6838$. (b) Phillips, A. J.; Uto, Y.; Wipf, P.; Reno, M. J.; Williams, D. R. Org. Lett. 2ooo, 2, 1165. (c) Petersson, M. J.; Jenkinsa, I. D.; A. Loughlin, W. A. Org. Biomol. Chem., 2oog, 7, 739.

(7) (a) Ishihara, M.; Togo, H. Tetrahedron 2007, 63, 1474. (b) Sayama, S. Synlett 2oo6, 1479. (c) Takahashi, S.; Togo, H. Synthesis 2oog, 2329. (d) Kangani, C. O.; Day, B. W. Tetrahedron Lett. 20o9, 50, 5332. (e) Cwik, A.; Hell, Z.; Hegedus, A.; Finta, Z.; Horvath, Z. Tetrahedron Lett. 2002, 43, 3985. (f) Wipf, P.; Wang, X. J. Comb. Chem. 2002, 4, 656. (g) Ilkgul, B.; Gunes, D.; Sirkecioglu, O.; Bicak, N. Tetrahedron Lett. 2010, 51, 5313.

(8) Mohammadpoor-Baltork, I.; Mirkhani, V.; Moghadam, M.; Tangestaninejad, S.; Zolfigol, M. A.; Abdollahi-Alibeik, M.; Khosropour, A. R.; Kargar, H.; Hojati, S. F. Catal. Commun. 2008, 9, 894.

(9) Mohammadpoor-Baltork, I.; Moghadam, M.; Tangestaninejad, S.; Mirkhani, V.; Hojati, S. F. Catal. Commun. 2008,9 , 1153.

(10) Mohammadpoor-Baltork, I.; Khosropour, A. R.; Hojati, S. F. Synlett 2005, 18, 2747.

(11) Mohammadpoor-Baltork, I.; Khosropour, A. R.; Hojati, S. F. Catal. Commun. 2oo7, 8, 200.

(12) Ge, H. X.; Liu, P.; Li, X. N.; Sun, W.; Li, J. L.; Yang, B. Q.; Shi, Z. Tetrahedron 2013, 69, 6591.

(13) Fu, G. C.; Lou, S. Org. Synth. 2010, 87, 310.

(14) Li, X. N.; Zhou, B. Y.; Zhang, J.; She, M. Y.; An, S. J.; Ge, H. X.; Li, C.; Yin, B.; Li, J. L.; Shi, Z. Eur. J. Org. Chem. 2012,8 , 1626.

(15) Wang, L.; Guo, B.; Li, H. -X.; Li, Q.; Lia, H. -Y.; Lang, J. -P. Dalton Trans. 2013, 42, 15570.

(16) Lazreg, F.; Slawin, A. M. Z.; Cazin, C. S. J. Organometallics 2012, 31, 7969.

(17) Bidal, Y. D.; Lazreg, F.; Cazin, C. S. J. ACS Catal. 2014, 4, 1564.

(18) Songis, O.; Boulens, P.; Benson, C. G. M.; Cazin, C. S. J. RSC Adv. 2012, 2, 11675.

(19) (a) Egbert, J. D.; Cazin, C. S. J.; Nolan, S. P. Catal. Sci. Technol. 2013, 3, 912. (b) Lesieur, M.; Lazreg, F.; Cazin, C. S. J.; Chem. Commun. 2014, 50, 8927 .

(20) Trose, M.; Lazreg, F.; Lesieur, M.; Cazin, C. S. J. Green Chem. 2015, 17, 3090.

(21) Santoro, O.; Collado, A.; Slawin, A. M. Z.; Nolan, S. P.; Cazin, C. S. J. Chem. Comm. 2013, 49, 10483.

(22) A mixture of mono and di-substitution results when the reaction is performed using less than 4 equiv. of the aminoalcohol.

(23) Uma Maheswari, C.; Sathish Kumar, G.; Venkateshwar, M. RSC Adv. 2014, 4, 39897. 
Graphical Abstract
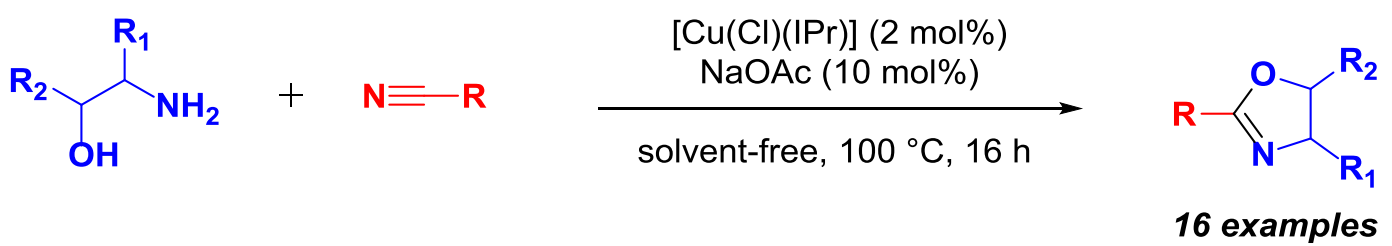